\title{
Our Understanding of the Universe (From Ancient to Present Time)
}

\author{
Dr. Vijay Mohan Das \\ University of God, Parade Ground, Fatehgarh, U.P., India
}

\begin{abstract}
Origin of the universe is still obscure. The reason being, one is not equipped with basic knowledge of structure of the matter, and atomic genetics as taught by a new science called participatory science. If nature breaks the matter, one would get its last and smallest particles called basic building blocks (B.B.Bs.) of which all fermions and bosons are composed. From these fermions and bosons all the matter of the universe is formed including human cell. These basic units are divine in the sense that they talk with each other by phenomenon called atomic transcription and translation. These are fundamental particles and atomic transcription and translation are fundamental working of the nature. These B.B.Bs. have power to transmutate to form bigger units of the universe like particles, atoms, molecules, cells, individuals, earth, solar system, galaxies etc. So, all effects of the universe are triggered by atomic transcription and translation or thought expressions. Before origin of the universe, these B.B.Bs. were in the form of tachyons. Out of the infinite tachyons one became the highest center of the universe. It had fed its thoughts to rest of B.B.Bs. that they would express only those thoughts to give desired effects as wished by highest center of the universe. Universe started with cold reaction. In this reaction cold dark matter was created. The density of the transformed universe before creation was low and the density of the CDM is very high. The density is defined by participatory science as number of basic building blocks per unit area. It would be discussed again in creation physics. So the space got vacated thus large volume of void was formed. Simultaneously in CDM layer by phenomenon of canalization, canals were formed and thus empty holes were there. At point $T$ hot reaction started with the result hydrogen was formed from tachyons. There liberated lot of energy during the creation and thus holes which were empty started ejecting huge radiations and pristine conditioned wispy hydrogen clouds and thus white holes or QSOS were formed in the nature. These ejected clouds which were nearer moved faster than those which were away from the CDM layer and thus Hubble law appeared in the universe. Universe kept on expanding with formation of more CDM by cold reaction all around and hydrogen clouds by hot reaction at point $T$ only. Void also kept on growing in size. After sometimes bright galaxies were formed by self gravitation. Early bright galaxies were very far from quasars that is why quasars are very distant object. Galaxies near the center of the universe are older while galaxies near the periphery are younger. Clouds at the edge are in pristine condition or they are just born ( 300000 years) from quasar. All points and the new model are towards an evolving universe not due to Big Bang rather due to creation which is still going inside quasars Thus our universe appeared into existence and all effects are triggered by atomic transcription or thought expressions. In atomic genetic engineering, (Technology more than speed of light) our B.B.B. talks with highest center of the universe via first transcription
\end{abstract}


to shift abnormal thought expressions to normal thought expressions. Thus the diseased cells could be transformed into normal cells leading to less complications in recovery.

Keywords: Basic Building Blocks : Atomic Genetics: Atomic Transcription and Translation: Tachyons and Atomic Genetic Engineering

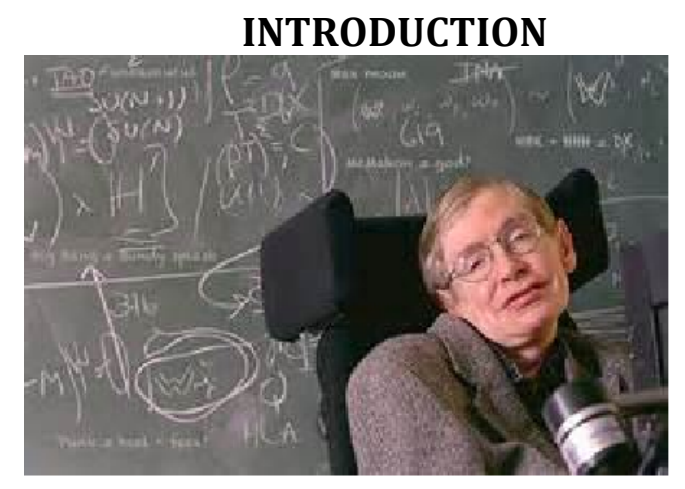

\section{PROF. S.W.HAWKING}

His hopes for the attainment of a fundamental theory of nature, and its relevance to the general public, are best summed up in the concluding paragraph of his famous book: "... if we do discover a complete theory, it should in time be understandable in broad principle by everyone, not just a few scientists. Then we shall all, philosophers, scientists and just ordinary people, be able to take part in the discussion of the question of why it is that the universe and we exist. If we find the answer to that, it would be the ultimate triumph of human reason, for then we would know the mind of God".

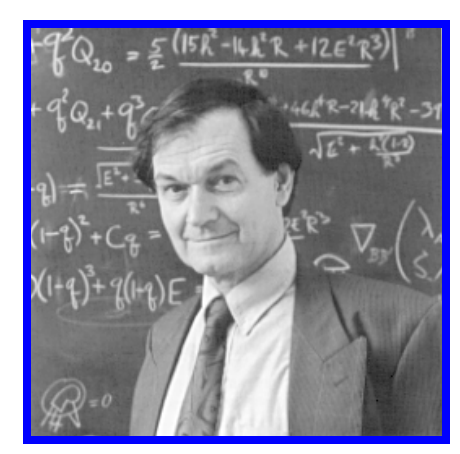

Prof. Roger Penrose- In the Emperor's New Mind, a bold brilliant, groundbreaking work, he argues that we lack a fundamentally important insight into physics, without which we will never be able to comprehend the mind. More over he suggests, insight may be the same one that will be required before we can write a unified theory of everything. 


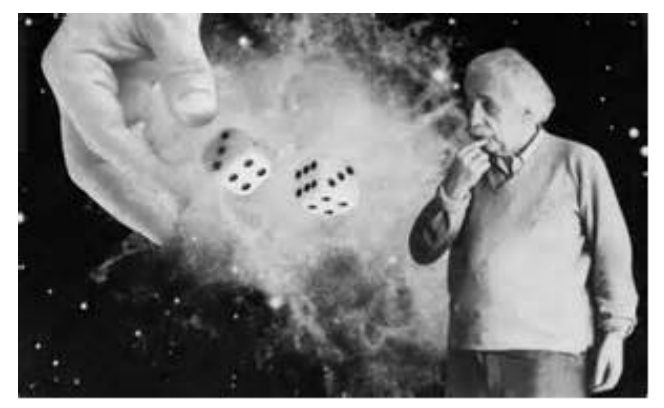

God does not play Dice

'I WANT TO KNOW HOW GOD CREATED THE UNIVERSE

I'M NOT INTERESTED IN THIS OR THAT PHENOMENON. I WANT TO KNOW HIS THOUGHTS. THE REST ARE DETAILS.

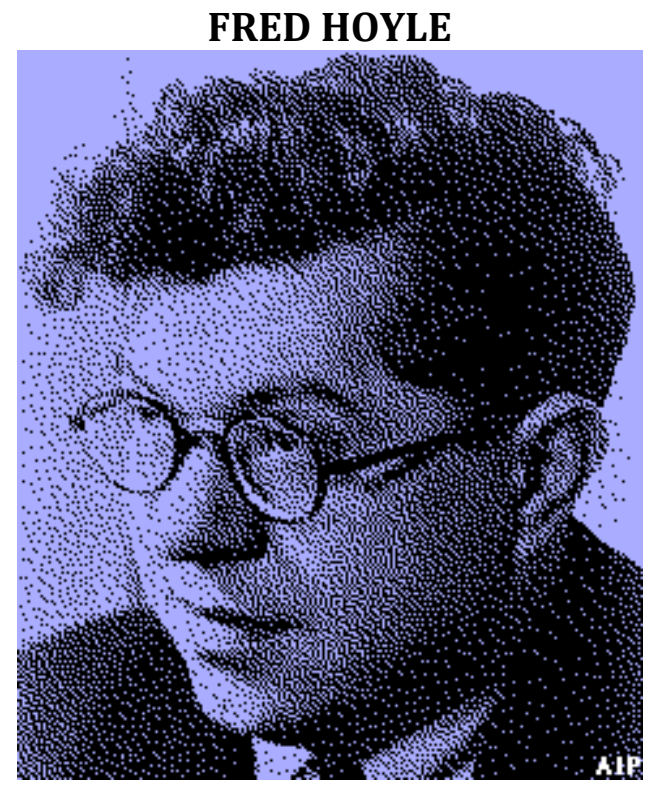

(1915 -2001)

Prof. Fred Hoyle had coined the word, "BIG BANG". He along with Prof. Harmann Bondi and Prof. Thomas Gold had introduced the concept of, "PERFECT COSMOLOGICAL PRINCIPLE" by the name steady state theory and $5 \%$ of the observations support this principle. Finally he along with Prof J.V.narlikar had given CONTINUOUS CREATION THEORY. According to Prof. Harmann Bondi, "It is $80 \%$ Big bang, $5 \%$ steady State and 15\% unknown". On this ground I have introduced a new model of the universe. This new model has capable to explain all the observations of Big bang (80\%), steady state observations (5\%) plus unknown observations (15\%). This NEW MODEL of the universe with new scientific understanding postulated by me that supports the continuous creation theory goes by the name of HOYLE -NARLIKAR UNIVERSE. [8]. 


\section{NEW INTERNATIONAL VERSION BIBLE - GENESIS 1}

Genesis [1] [1] 1 - In the beginning God created the heavens and the earth. Now the earth was formless and empty, darkness was over the surface of the deep, and the Spirit of God was hovering over its waters.

\section{FLAT-EARTH UNIVERSE}

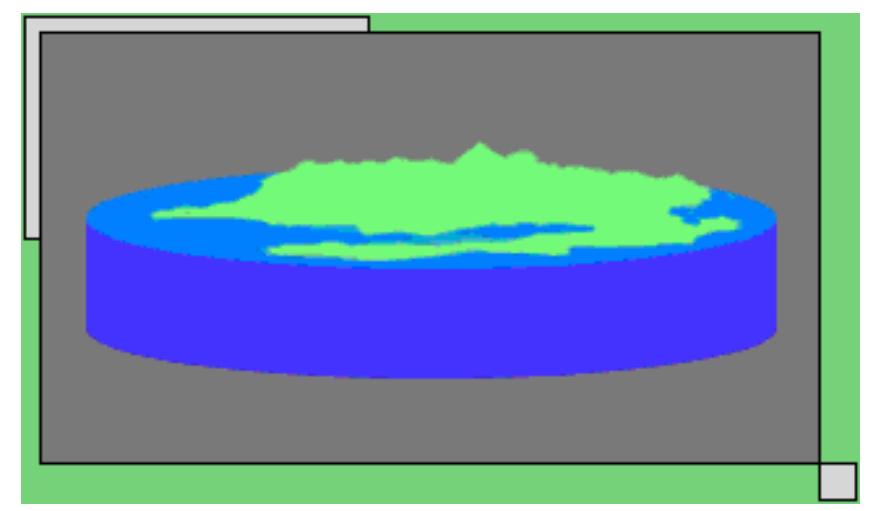

Not so much a theory of the universe as a simple picture of the planet we call home, the flatearth model proposed that Earth's surface was level. Although everyday experience makes this seem a reasonable assumption, direct observation of nature shows the real world isn't that simple. For instance, when a sailing ship heads into port, the first part that becomes visible is the crow's-nest, followed by the sails, and then the bow of the ship. If the Earth were flat, the entire ship would come into view at once as soon as it came close enough to shore.

The Greek philosopher Aristotle provided two more reasons why the Earth was round. First, he noted that Earth's shadow always took a circular bite out of the moon during a lunar eclipse, which would only be possible with a spherical Earth. (If the Earth were a disk, its shadow would appear as an elongated ellipse at least during part of the eclipse.) Second, Aristotle knew that people who journeyed north saw the North Star ascend higher in the sky, while those heading south saw the North Star sink. On a flat Earth, the positions of the stars wouldn't vary with a person's location.

Despite these arguments, which won over most of the world's educated citizens, belief in a flat Earth persisted among many others. Not until explorers first circumnavigated the globe in the 16th century did those beliefs begin to die out. Yet a diehard core of flat-earth believers persisted even past the days of the Apollo Moon missions and those glorious images of a spherical Earth suspended against the blackness of space.

\section{PTOLEMY'S UNIVERSE}

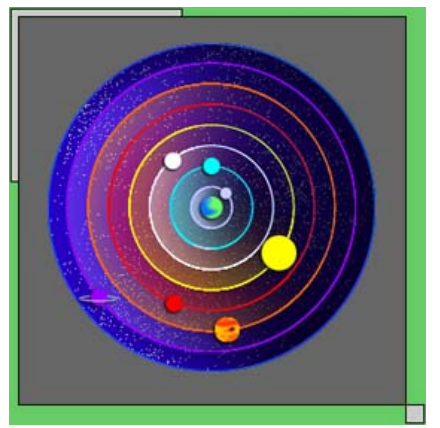


Ptolemy placed Earth at the center of the universe, with the Moon, Mercury, Venus, the Sun, Mars, Jupiter, and Saturn circling our planet.

For most ancient astronomers, accurately predicting the positions of the planets was tantamount to understanding the workings of the universe. The far more distant stars were simply the backdrop against which planetary action took place. Ptolemy, the last of the great Greek astronomers of antiquity, developed an effective system for mapping the universe. Basing much of his theory on the work of his predecessor, Hipparchus, Ptolemy designed a geocentric, or Earth-centered, model that held sway for 1400 years.

That Ptolemy could place Earth at the center of the universe and still predict the planets' positions adequately was a testament to his ability as a mathematician. That he could do so while maintaining the Greek belief that the heavens were perfect-and thus that each planet moved along a circular orbit at a constant speed-is nothing short of remarkable.

The greatest difficulties he had to overcome were explaining the changing speeds and the occasional east-to-west, or retrograde, motion of the planets. He accomplished this by having each planet move along a small circle, called an epicycle, whose center travelled along a larger circle, called a deferent, with Earth at its center.

Although this scheme came close to accomplishing what he wanted, it still came up a little short. So Ptolemy made a couple of refinements. First, he placed Earth slightly away from the center of the deferent. (A slightly off-center circle comes very close to mimicking an ellipse.) And second, he had the center of the epicycle move at a constant angular speed around a third point, called the equant, which lay on the opposite side of the deferent's center from Earth. These modifications allowed Ptolemy to predict the positions of the planets with reasonablethough far from perfect-accuracy.

\section{COPERNICUS' UNIVERSE}

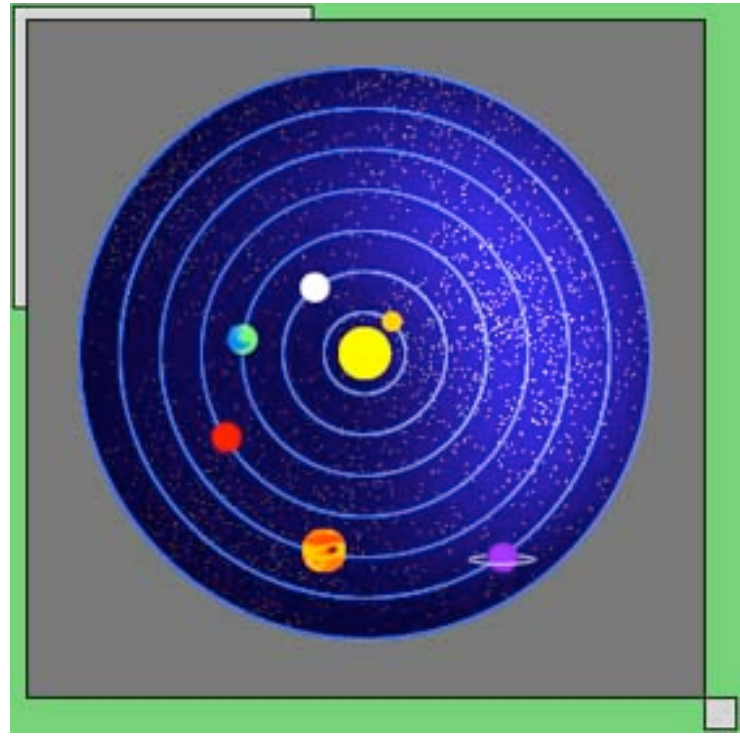

The Sun was Copernicus' center of the universe, encircled by Mercury, Venus, Earth, Mars, Jupiter, and Saturn.

Copernicus made a great leap forward by realizing that the motions of the planets could be explained by placing the Sun at the center of the universe instead of Earth. In his view, Earth 
was simply one of many planets orbiting the Sun, and the daily motion of the stars and planets were just a reflection of Earth spinning on its axis. Although the Greek astronomer Aristarchus developed the same hypothesis more than 1500 years earlier, Copernicus was the first person to argue its merits in modern times.

In Copernicus' Sun-centered (heliocentric) view of the cosmos, the planets' occasional backward, or retrograde, motion comes about naturally through the combined motions of Earth and the planets. As Earth speeds around the Sun in its faster orbit, it periodically overtakes the outer planets. Like a slower runner in an outside lane at a track meet, the more distant planet appears to move backward relative to the background scenery.

Copernicus' model also explains why the two planets closest to the sun, Mercury and Venus, never stray far from the Sun in our sky. And it allowed Copernicus to calculate the approximate scale of the solar system for the first time. That's not to say Copernicus' model was without problems: He still clung to the classical idea that the planets should move in circular orbits at constant speeds, so like Ptolemy, he had to jury-rig a system of circles within circles to predict the planets' positions with reasonable accuracy.

Despite the basic truth of his model, Copernicus did not prove that Earth moved around the Sun. That was left for later astronomers. The first direct evidence came from Newton's laws of motion, which say that when objects orbit one another, the lighter object moves more than the heavier one. Because the Sun has about 330,000 times more mass than Earth, our planet must be doing almost all the moving. A direct observation of Earth's motion came in 1838 when the German astronomer Friedrich Bessel measured the tiny displacement, or parallax, of a nearby star relative to the more distant stars. This minuscule displacement reflects our planet's changing vantage point as we orbit the Sun during the year.

\section{KEPLER'S UNIVERSE}

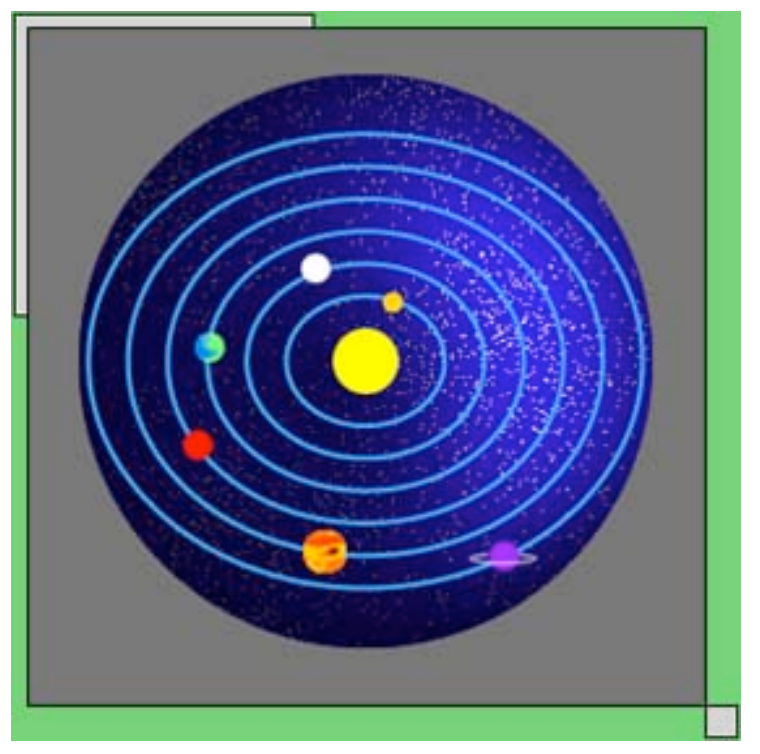

Kepler modified Copernicus' model by moving the planets in elliptical, rather than circular, orbits. Kepler took Copernicus' heliocentric view of the universe and removed the requirement that the planets move in circular orbits at constant speeds. But that was only after he exhausted every combination of circular motions he could conceive. 
Basing his work on the meticulous and exceedingly accurate naked-eye observations of the Danish astronomer Tycho Brahe, Kepler tried for more than a decade to match the positions of Mars to some sort of circular motion. Only after he ran out of possibilities did he try to fit the observations with another type of curve called an ellipse, the next-simplest form after the circle. He found that the positions of Mars matched almost perfectly with an elliptical path, and that the other planets followed suit.

This became the first of his three laws of planetary motion. He next tackled the problem of the planets' varying speeds. He determined that a planet travels most rapidly when it comes closest to the Sun and moves slowest when farthest away. His third and final law of planetary motion gives the precise relation between the distance of a planet from the Sun and how fast it completes an orbit.

\section{STEADY-STATE UNIVERSE}

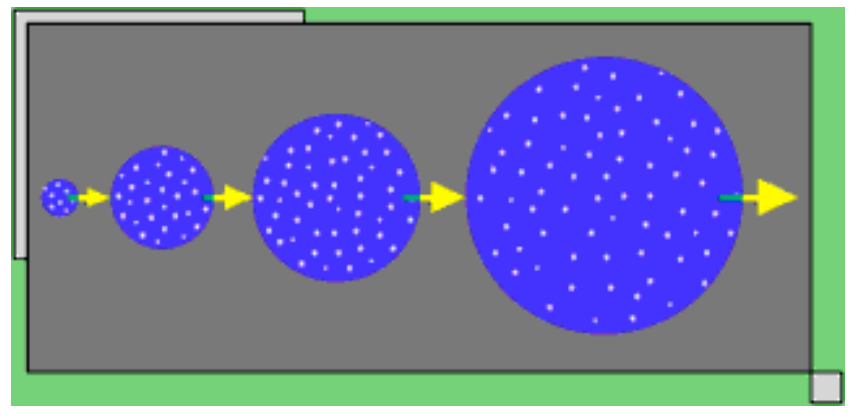

Proposed in 1948 by Hermann Bondi, Thomas Gold, and Fred Hoyle, the steady-state theory was based on an extension of something called the perfect cosmological principle. This holds that the universe looks essentially the same from every spot in it and at every time. (This applies only to the universe at large scales; obviously planets, stars, and galaxies are different from the space between them.)

Obviously, for the universe to look the same at all times, there could have been no beginning or no end. This struck a philosophical chord with a number of scientists, and the steady-state theory gained many adherents in the 1950s and 1960s. How could the universe continue to look the same when observations show it to be expanding, which would tend to thin out its contents? Supporters of this cosmology balanced the ever-decreasing density that results from the expansion by hypothesizing that matter was continuously created out of nothing. The amount required was undetectably small-about a few atoms for every cubic mile each year.

The steady-state theory began to wither in the 1960s. First, astronomers discovered quasars, the highly luminous cores of very distant galaxies. Because the vast majority of quasars lie exceedingly far away, their existence proves that the perfect cosmological principle cannot be true-the distant and therefore ancient universe is not the same as the younger universe nearby. The death knell for the theory sounded when radio astronomers Arno Penzias and Robert Wilson discovered the cosmic microwave background, the leftover radiation from the Big Bang. The steady-staters had no reasonable way to explain this radiation, and their theory slowly faded away as so many of its predecessors had. 


\section{BIG BANG UNIVERSE}

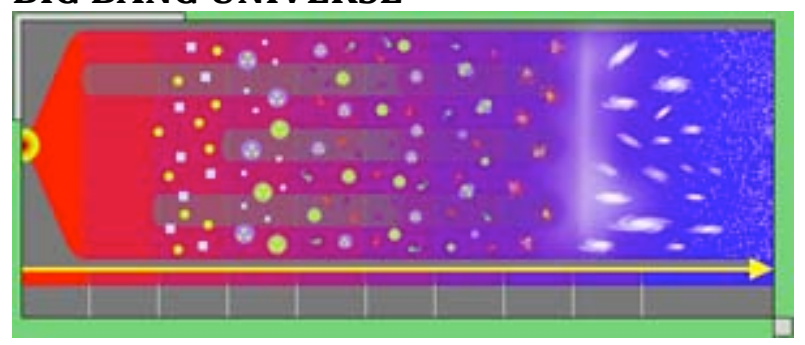

How did the universe really begin? Most astronomers would say that the debate is now over: The universe started with a giant explosion, called the Big Bang. The big-bang theory got its start with the observations by Edwin Hubble that showed the universe to be expanding. If you imagine the history of the universe as a long-running movie, what happens when you show the movie in reverse? All the galaxies would move closer and closer together, until eventually they all get crushed together into one massive yet tiny sphere. It was just this sort of thinking that led to the concept of the Big Bang.

The Big Bang marks the instant at which the universe began, when space and time came into existence and all the matter in the cosmos started to expand. Amazingly, theorists have deduced the history of the universe dating back to just 10-43 second (10 million trillion trillion trillionths of a second) after the Big Bang. Before this time all four fundamental forcesgravity, electromagnetism, and the strong and weak nuclear forces-were unified, but physicists have yet to develop a workable theory that can describe these conditions.

During the first second or so of the universe, protons, neutrons, and electrons-the building blocks of atoms-formed when photons collided and converted their energy into mass, and the four forces split into their separate identities. The temperature of the universe also cooled during this time, from about 1032 (100 million trillion trillion) degrees to 10 billion degrees. Approximately three minutes after the Big Bang, when the temperature fell to a cool one billion degrees, protons and neutrons combined to form the nuclei of a few heavier elements, most notably helium.

The next major step didn't take place until roughly 300,000 years after the Big Bang, when the universe had cooled to a not-quite comfortable 3000 degrees. At this temperature, electrons could combine with atomic nuclei to form neutral atoms. With no free electrons left to scatter photons of light, the universe became transparent to radiation. (It is this light that we see today as the cosmic background radiation.) Stars and galaxies began to form about one billion years following the Big Bang, and since then the universe has simply continued to grow larger and cooler, creating conditions conducive to life.

Three excellent reasons exist for believing in the big-bang theory. First, and most obvious, the universe is expanding. Second, the theory predicts that 25 percent of the total mass of the universe should be the helium that formed during the first few minutes, an amount that agrees with observations. Finally, and most convincing, is the presence of the cosmic background radiation. The big-bang theory predicted this remnant radiation, which now glows at a temperature just 3 degrees above absolute zero, well before radio astronomers chanced upon it. 


\section{FRIEDMANN UNIVERSE}

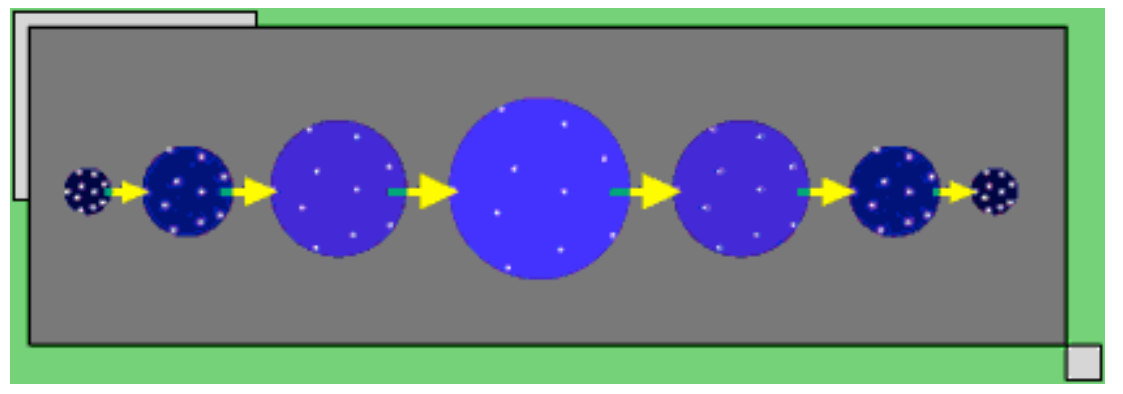

Closed Universe: The Big Bang's momentum is offset by gravity, producing a "Big Crunch."

In the early 1920s, Russian physicist and mathematician Alexander Friedmann became the first person to embrace the idea that the equations of Einstein's general theory of relativity called for a universe in motion. Einstein (and most other scientists, for that matter) believed that the universe was static, and he modified his equations by including a "cosmological constant" to keep it so.

Friedmann made two simple assumptions about the universe: that when viewed at large enough scales, it appears the same both in every direction and from every location. From these assumptions (called the cosmological principle) and Einstein's equations, he developed the first model of a universe in motion. The Friedmann universe begins with a Big Bang and continues expanding for untold billions of years—that's the stage we're in now. But after a long enough period of time, the mutual gravitational attraction of all the matter slows the expansion to a stop. The universe then starts to fall in on itself, replaying the expansion in reverse. Eventually all the matter collapses back into a singularity, in what physicist John Wheeler likes to call the "Big Crunch".

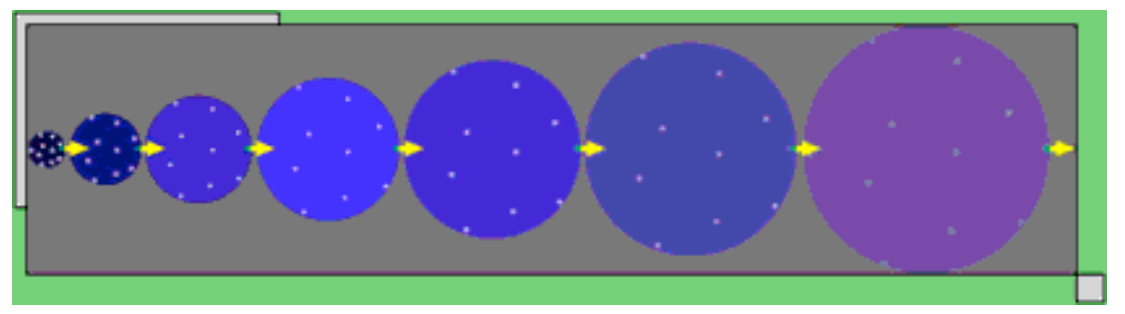

Open Universe: There is not enough matter to stop the universe from expanding forever

Although Friedmann found only this one solution, called a closed universe because the size of the universe is finite, two similar solutions exist. In an open universe, there's not enough matter to bring the expansion to a halt. Galaxies continue to separate from one another, although more slowly as time passes. Eventually all the stars go out, and the universe becomes cold and dark. Intermediate between the open and closed universes is the flat universe. In this case, the universe expands forever, but the speed at which the galaxies separate eventually approaches zero. What kind of universe do we live in? Observations of the universe's density should eventually tell us, but they are not yet accurate enough to distinguish among the three possibilities 


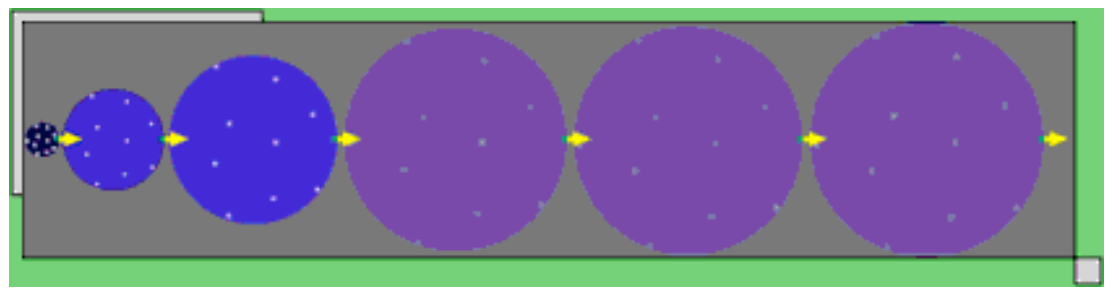

Flat Universe: Expansion slows until the rate approaches zero.

\section{ANTHROPIC UNIVERSE}

Why is the universe the way it appears? Some scientists think that our very existence provides the answer. To them, many of the physical properties of the universe seem finely tuned for producing life. For instance, if the relative strengths of the four fundamental forces were slightly different, stars might never have formed and life as we know it would have been impossible. Or if the universe had expanded slightly faster than it did, matter would have spread out too quickly to coalesce into any significant objects. Conversely, if the expansion had been just a little slower, the universe would have already collapsed back into a "Big Crunch."

These and other cosmic "coincidences" led some scientists to speculate that the universe is the way it is because we are here to observe it. This anthropic principle has two basic versions, the weak and the strong. The weak version, developed by Robert Dicke in the early 1960s, states that in a large universe, intelligent life can exist only during a narrow window of time. We shouldn't be surprised at the universe we see because we could never be around to view it at a significantly different time.

The strong anthropic principle goes much further. Proposed by Brandon Carter in the late 1960s, it states that among all the possible universes that could exist, only a special few have the right conditions that could give rise to intelligent life. The cosmic coincidences are then not some fundamental aspect of the way the laws of physics operate, but rather a prerequisite for the development of life. If the strong anthropic principle is true, then some would argue that the universe was designed with a purpose. If it is false, then a future "theory of everything" should be able to explain why the seeming coincidences that created life really are not. 


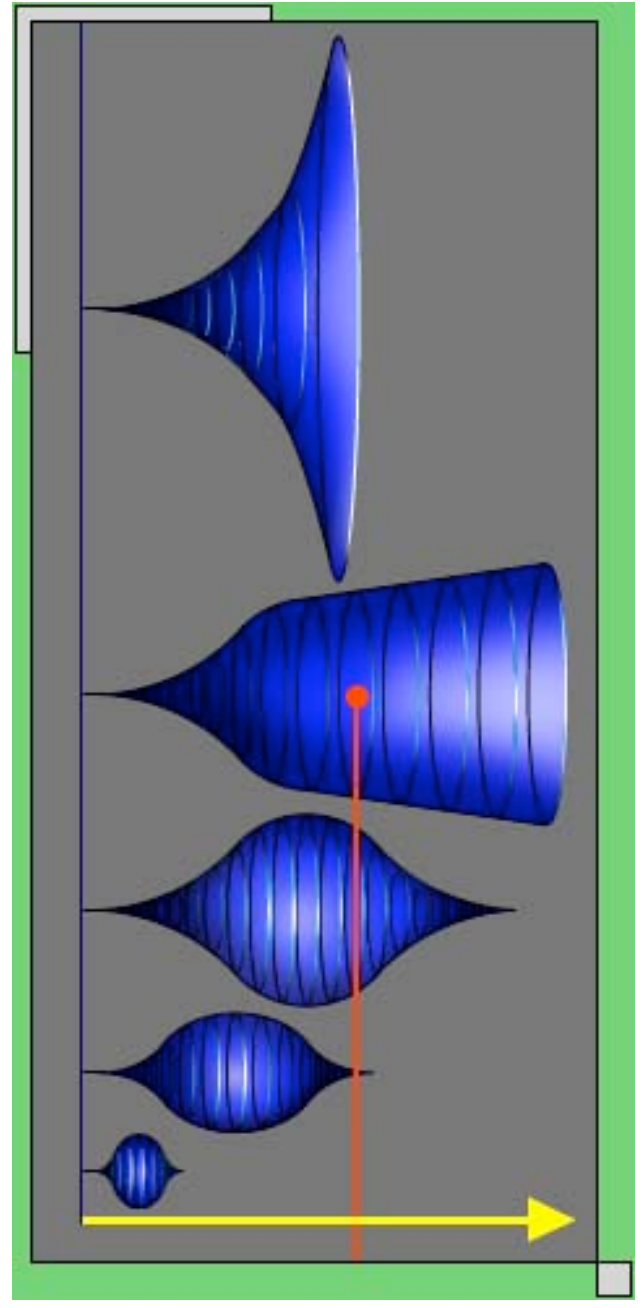

While other universes either expand too quickly and flatten (top) or close before life can evolve (bottom three), our universe (second from top) seems perfectly poised to support life.

\section{INFLATIONARY UNIVERSE}

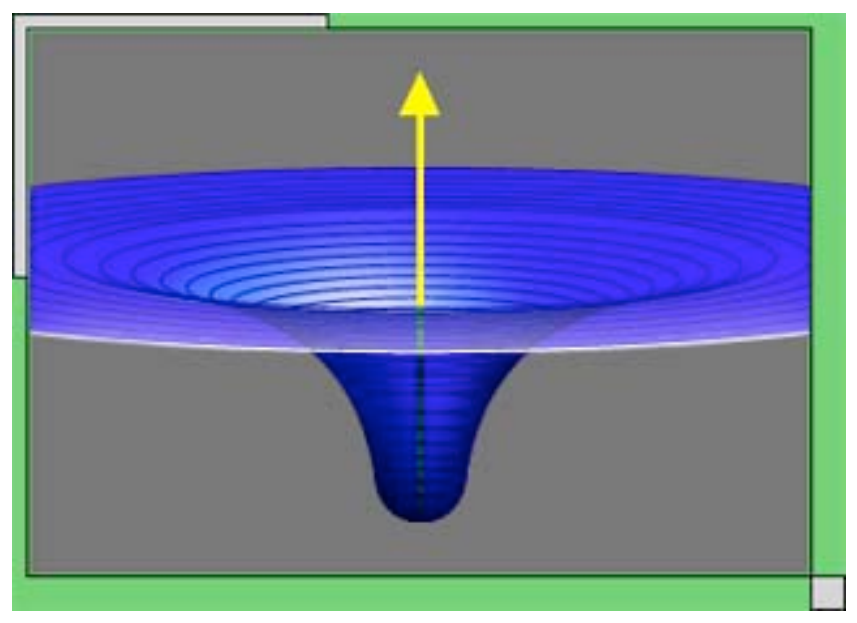

An extraordinary burst of expansion in the very early stages of the universe inflated the size of the cosmos by a factor of 1050 . This contrasts with the standard big-bang model, which has the universe expanding at an ever-decreasing rate as gravity tries to pull all the matter back together. 
The big-bang theory does a remarkable job of describing the universe we see today: It explains the expansion of the universe, predicts the correct abundances of hydrogen and helium (the most common elements in the universe), and accounts for the cosmic background radiation. Few scientists today doubt its validity.

Despite its successes, the standard big-bang theory was too simple to be complete. For example, it offered no reason why the temperature of the background radiation remains remarkably constant over the entire sky, varying by no more than one part in 100,000 . In the standard big-bang model, the constituents of the early universe could not all interact with one another, so there was no way for them all to reach the same temperature. Another problem is that the universe appears very nearly flat, existing right on the knife edge between being open and closed. In the standard big-bang model, the only way to explain these observations is to have the universe start out with a uniform temperature and at the critical density.

In 1980, the American physicist Alan Guth devised a way around these problems. He theorized that shortly after the Big Bang (10-35 seconds, or 100 billion trillion trillionths of a second, to be exact), the universe underwent a period of extraordinarily rapid expansion, inflating its size by a factor of 1050 .

Before this inflationary period, the universe's constituents would have been in contact with one another, so they would have reached the same temperature. And the rapid inflation would make the universe's expansion appear very flat, in the same way that the surface of a balloon blown up by such a huge factor would resemble the Great Plains. Inflation ended by 10-30 seconds after the Big Bang, and since then the universe has expanded just as it would have in the standard big-bang model.

Guth based his argument on the Grand Unified Theories, or GUTs, that unite gravity, electromagnetism, and the weak and strong forces into one. These theories predict that as the universe cooled after the Big Bang, the forces separated into their individual identities at what are called phase transitions. Water undergoes a similar phase transition when it freezes into ice as the temperature drops. If conditions are right, you can supercool water below the freezing point without ice forming. If the universe behaved similarly, then space would have had an excess energy that counteracted gravity, driving the inflation.

\section{NO-BOUNDARY UNIVERSE}

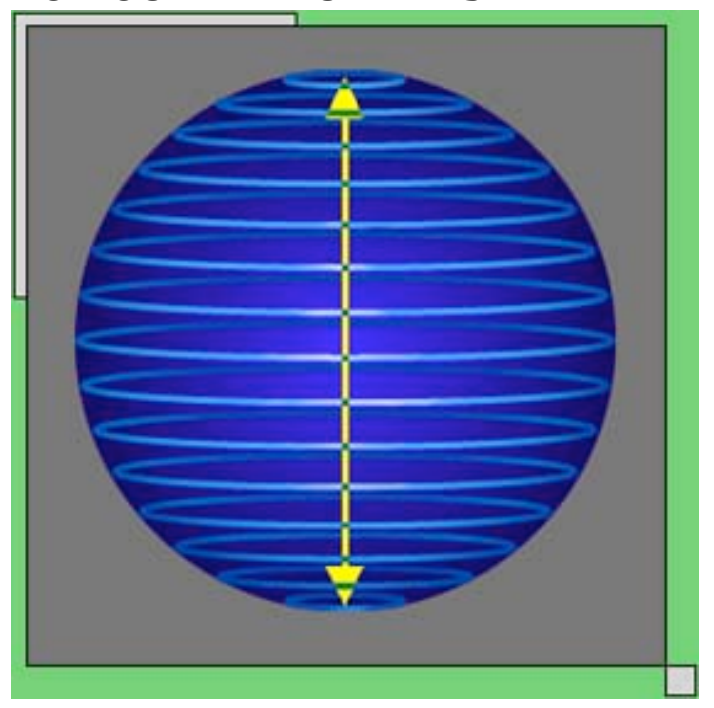


A universe that is finite in size but did not begin with a singularity is the result of one attempt to combine aspects of general relativity and quantum mechanics. The history of this noboundary universe in imaginary time is like the surface of Earth, with the Big Bang equivalent to Earth's North Pole and the size of the universe increasing with imaginary time as you head south toward the equator.

A proposal first advanced by Stephen Hawking and Jim Hartle, the no-boundary universe is one in which the universe does not start with a singularity. It uses American physicist Richard Feynman's proposal to treat quantum mechanics as a "sum over histories," meaning that a particle does not have one history in space-time but instead follows every possible path to reach its current state. By summing these histories-a difficult process that must be done by treating time as imaginary-you can find the probability that the particle passes through a particular point.

Hawking and Hartle then wedded this idea to general relativity's view that gravity is just a consequence of curved space-time. Under classical general relativity, the universe either has to be infinitely old or had to have started at a singularity. But Hawking and Hartle's proposal raises a third possibility - that the universe is finite but had no initial singularity to produce a boundary (thus the name).

The geometry of the no-boundary universe would be similar to the geometry of the surface of a sphere, except it would have four dimensions instead of two. You can travel completely around Earth's surface, for instance, without ever running into an edge. In this analogy, unfolding in imaginary time, Earth's North Pole represents the Big Bang, marking the start of the universe. (But just as the North Pole is not a singularity, neither is the Big Bang).

\section{OSCILLATING UNIVERSE}

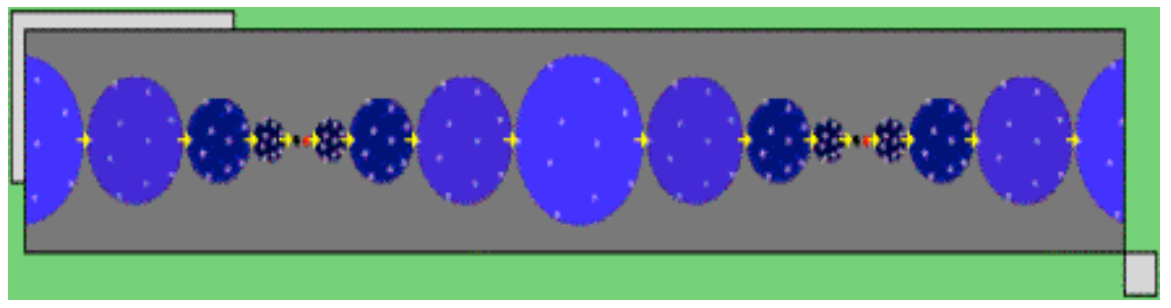

One of the implications of the big-bang theory is that the universe will one day end, or at least any life in the universe will come to an end. If the universe is either open or flat, meaning that it expands forever, it will survive for an infinite period of time. But eventually all the material in all the generations of stars will be exhausted, and the universe will grow cold and dark. In a closed universe, in which the expansion eventually stops and a contraction follows, the end is far from cold and dark-as the Big Crunch approaches, the universe grows hotter and brighter until it implodes into a singularity and gets crushed out of existence.

But is that what would really happen? Some scientists speculate that the Big Crunch would not signal the end. Perhaps another Big Bang would follow the Big Crunch, giving rise to a new universe of possibilities. The idea that Bangs follow Crunches in a never-ending cycle is known as an oscillating universe. Though no theory has been developed to explain how this could ever happen, it has a certain philosophical appeal to people who like the idea of a universe without end. 


\section{HOYLE- NARLIKAR UNIVERSE}

\section{NEW MODEL OF THE UNIVERSE AND NEW SCIENTIFIC UNDERSTANDING}

Hoyle and Narlikar proposed (in their continuous creation theory) that new matter is being created due to 'IMPLOSION' to balance the expansion of the universe, which astronomers have observed. Inside 'QUASI-STARS' gravitational collapses may form some matter in the universe. The huge luminosity and the radio emission from these quasi-stars appear to be 'gravity powered' unlike ordinary stars, which derive their energy from nuclear reactions.

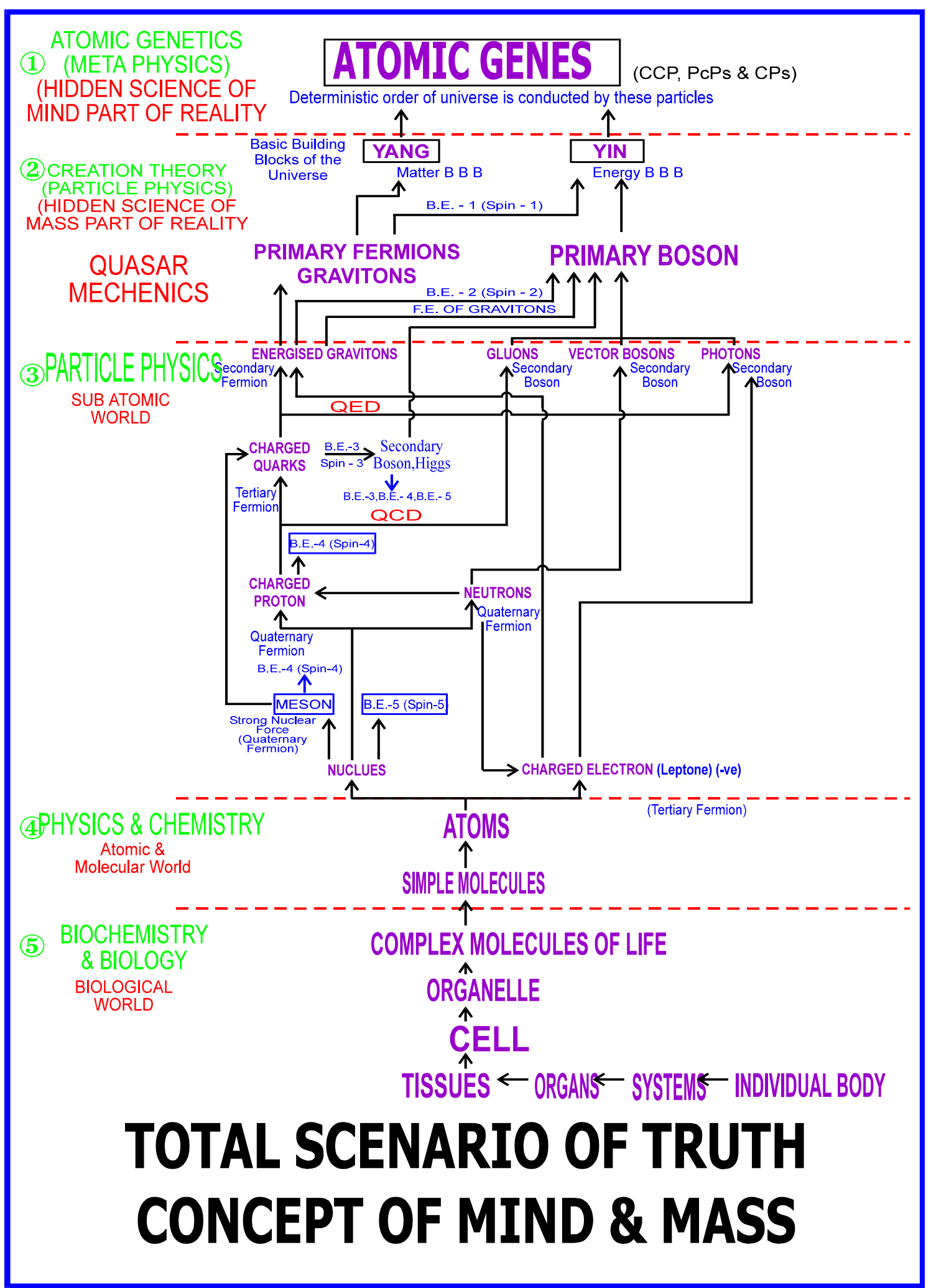




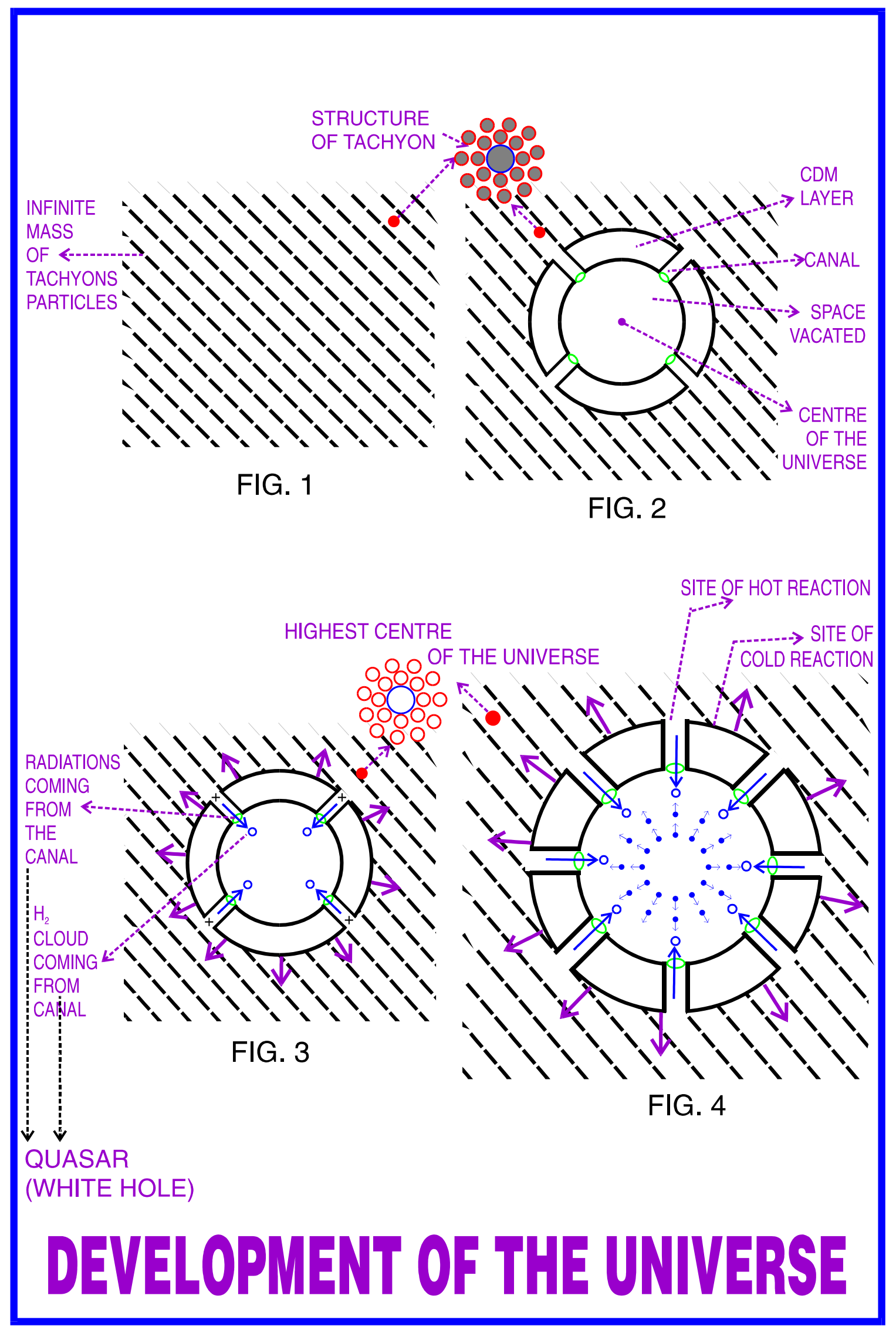




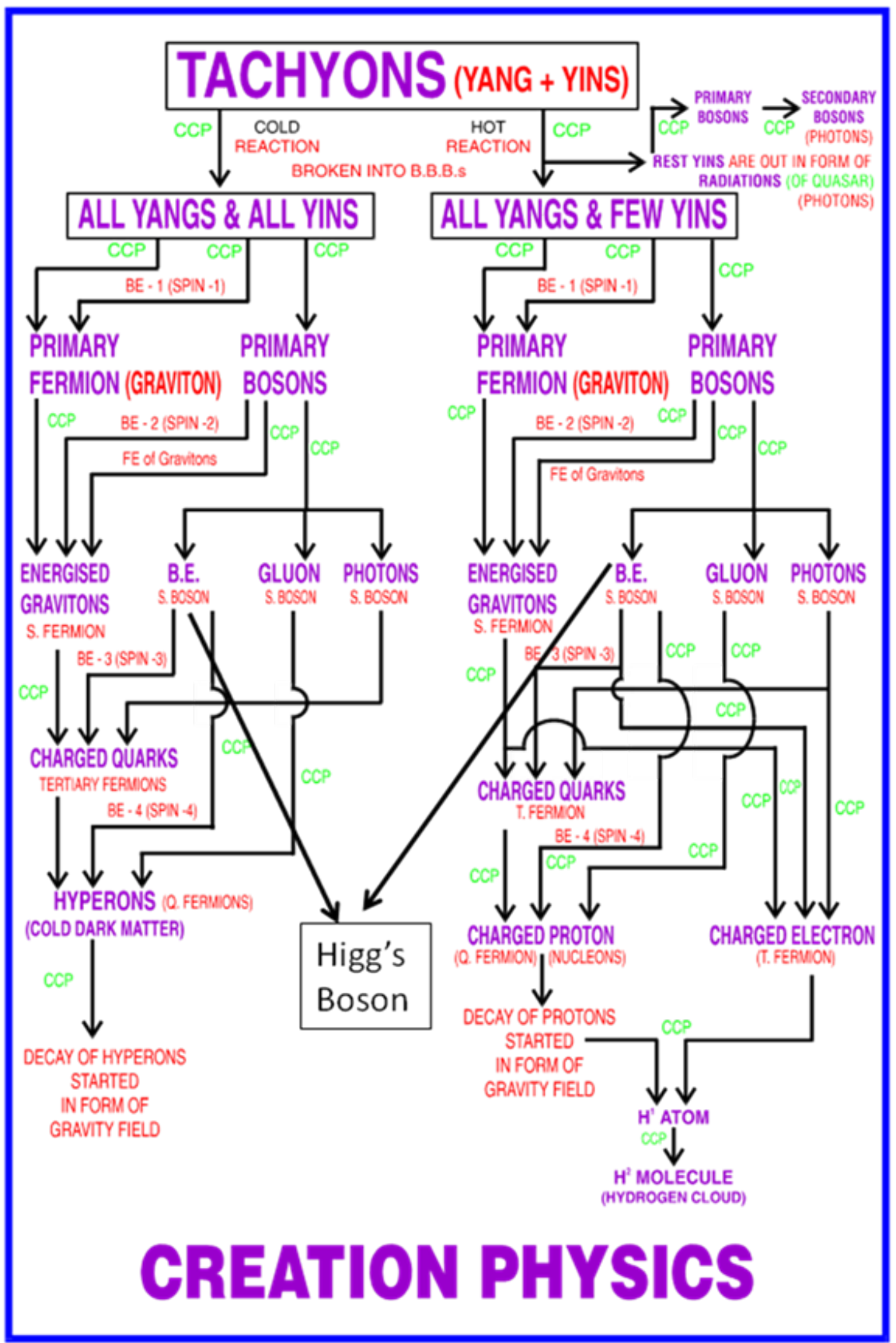




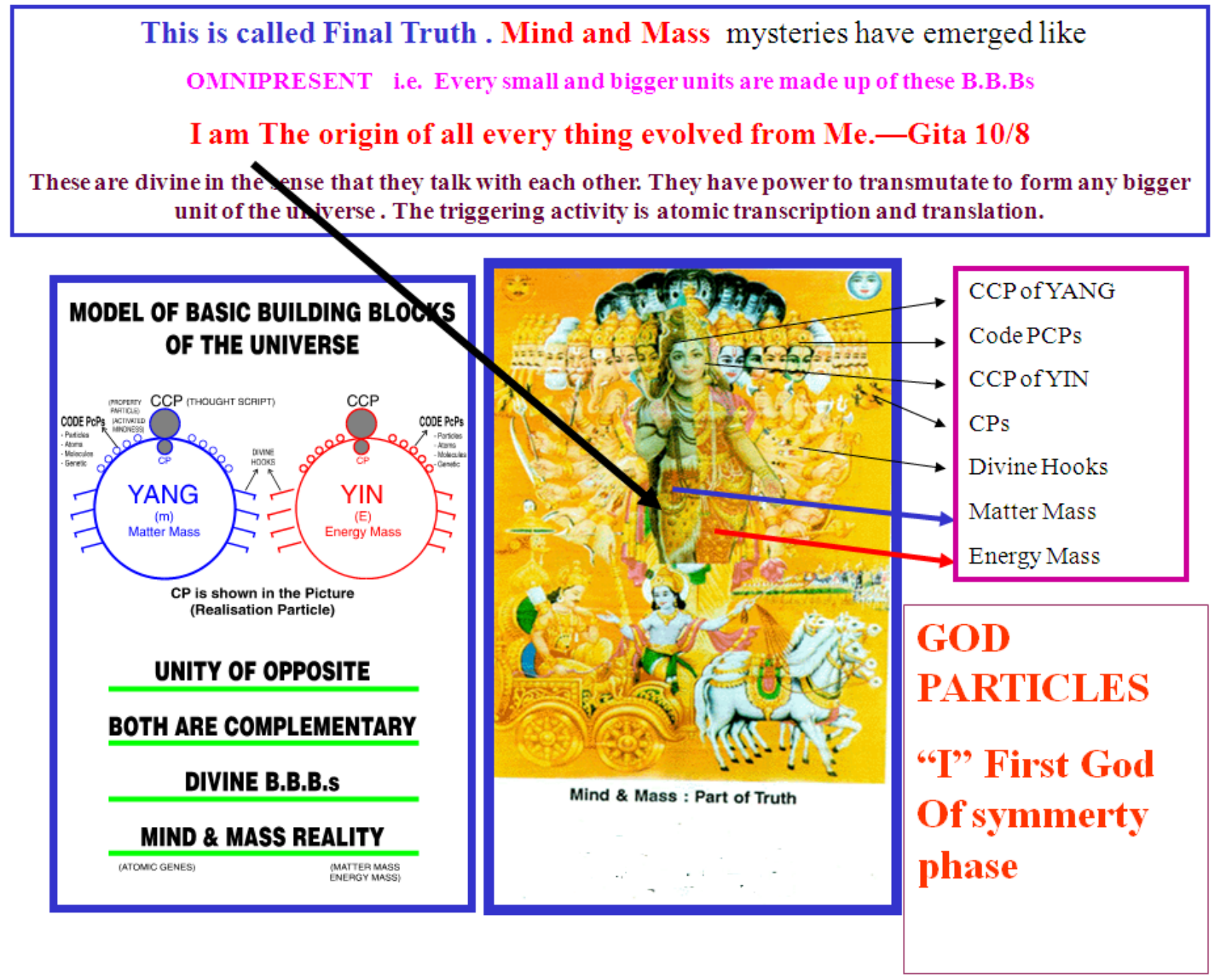

(Fig 4- Science without Religion is blind and Religion without Science is Lame- Albert Einstein)

Before the origin of the universe, these Basic Building Blocks (B.B.Bs) (Fig-1 and Fig 4) were in the form of tachyons (Fig-2). It means that at that time the tachyons were everywhere in the universe. Let us look at the structure of tachyons; it is made up of one matter B.B.B. (YANG) and many energy (YINs) B.B.Bs. Initially out of the infinite tachyons, one became the highest center of the universe. Messages used to go from highest center to rest of the universe and messages could come from rest of the universe to highest center of the universe by atomic transcription. Thus highest center had fed its thought to rest of the B.B.Bs. that would take part in creation - that they would express only those thoughts to give desired effect as wished by the highest center of the universe. So all B.B.Bs were informed about their role before creation of the universe. In pre-creation era programming of the future universe was done by highest center of the universe.

Our universe is oscillating and it is a divine universe. It means that it has a creation phase and a destruction phase. During creation phase tachyons break into their B.B.Bs. and from these B.B.Bs, formation of fermions and bosons take place (Fig-3). After the creation phase, destruction would start and in this phase all created particles would again break into their B.B.Bs and finally tachyons would form.

At the time of origin of the universe, all the effects got created. These effects are taking of different shapes and appearance of properties and law. All these effects are studied in various branches of science. 
With the origin of the universe, nature first created a sphere of COLD DARK MATTER (C.D.M) and canals in it. With the result space got created. At the other end of the canals, hot reaction started (the relics are back ground radiations 2.7 degree $\mathrm{K}$ of our hydrogen clouds). As a result hydrogen clouds and lot of radiations were created. The empty canals were filled by these hydrogen clouds and radiations and thus QUASARS appeared in the universe. Simultaneously C.D.M. layer started expanding and clouds and radiations kept on coming in this closed universe (Fig-2). With the passage of time more and more C.D.M. layer formed, more and more quasars formed. The hydrogen cloud came in this closed universe. They started running towards C.D.M. layer as they were attracted by the gravity of C.D.M. layer. Those clouds, which were nearer, moved faster than those, which were away from CDM Layer. The HUBBLE LAW, can thus be explained. With some more passage of time, clouds were joined to form GMC (giant molecular clouds). Later by self-gravitation different proto stars, proto planets, proto satellites were formed. Finally stars became bright and thus bright galaxies appeared in this universe. Our universe is still in expansion phase and creation is still going inside quasars. It is to be remembered that highest center of the universe does not come in the visible universe. It keeps on receiving the messages by atomic transcription and it has power to change any programming programmed by it during pre-creation era.

\section{MOTIVATION}

Prof. J.V. Narlikar and Prof. Fred Hoyle had proposed continuous creation theory [1] in 1960s. The new model of the universe made by participatory science which is contrary to Big bang model supports theory proposed by Prof. Narlikar and Prof. Hoyle with addition of new scientific understanding calling it a "NEW PHYSICS". Prof. Hermann Bondi, co-author of Steady State theory said, "It is $80 \%$ big bang, $5 \%$ steady state and $15 \%$ unknown". New model of the universe not only explains all the events of Big bang and steady state but also $15 \%$ unknown events i.e. quasars and cold dark matter which is constituting $90 \%$ to $98 \%$ of the matter of the universe. Researches regarding structure of the matter are held up at lepto-quark level. Prof. J.V.Narlikar had asked to investigate the structure of the matter beyond leptons and quarks (the ultimate structure of the matter) in an article titled," Do Astronomical observation Require New Physics?” in Physics News, Vol-30, N0-3\&4, Sept \&Dec, 1999 [2]. New Scientific understanding has explored the matter beyond lepto-quarks and ultimate structure of the matter i.e. Basic Building Blocks ( B.B.Bs) are not only hypothesized but also there are observations that confirm their existence. Mathematics as well as Experimental Labs are required to know structure of the matter up to the level of leptoquarks. Beyond that it is the participatory science discipline which is required to know structure of the matter up to the level of Basic Building Blocks. As we have radioactivity, where nature is breaking itself to know about structure of the nucleus of the atom. Without this breaking it is not possible to study about nucleus. Similarly nature is breaking itself beyond leptoquark up to the level of Basic Building Blocks in the universe, only we have to re-explain those observations in terms of their constituents. These are-

1) Proton Decay - As we observe decay of nucleus in radioactivity into alpha, beta and gamma, similarly proton does decay forming gravity and electromagnetic field particles. These field particles - gravitons (secondary fermions) are coming out from quarks while photons (secondary bosons) are coming out from decay of gravitons.

2) Gravity observations - all gravity interactions should be re-explained. During these interactions gravitons interact by breaking themselves. By these observations we could know structure of the matter of secondary fermions and secondary bosons up to the level of primary fermions and primary boson.

3) Quasar observations - Inside quasar Nature is breaking itself up to the level of Basic Building Blocks (CREATION PHYSICS). So we could see the basic constituents of all the 
force particles (except weak nuclear force which is mediated by vector bosons) We could see up to the level of Basic Building blocks (B.B.Bs)

4) Our Brain realisation - Inside our brain nature is working by breaking its last box i.e. atomic genes. Breaking of atomic genes, which is the property of the matter or basic building blocks, is triggering the thought process and other working of the brain. We could see up to the level of atomic genes property of Basic Building blocks.

Participatory science is a new discipline in science as proposed by Prof. John A. Wheeler [3]. Prof. John Wheeler sees this involvement of the observer as the most important feature of the quantum theory and he has therefore suggested replacing the word 'observer' by the word 'participator'. The idea of participation instead of observation has been formulated in modern physics only recently. Modern science teaches us up to the level of lepto-quarks. Beyond that, it is the participatory science that teaches us about structure of the matter upto the level of Basic Building Blocks (B.B.Bs) i.e. ultimate structure of the matter of which all fermions and bosons are composed. The entire participatory science has been developed by me and we could see structures which are beyond our visibility i.e. both macro (invisible universe) and micro (ultimate structure of the matter) worlds. Big bang and steady state models have been made because we could see only $30 \%$ of visible universe. Rest of visible and invisible universe could be seen through participatory science while making new model of the universe. Till today no attempt has been made to investigate about the consciousness of the matter. D. Bohm has found it is necessary to regard consciousness as an essential feature of the holomovement and it should be taken into account explicitly while considering this theory. He sees mind and matter as being interdependent and correlated but not causally connected [4]. Atomic genetics, a new concept in science has been introduced. It is the study as regard mind part of the reality. There are observations that show that matter is related with consciousness. The most exciting observation is expansion of the universe shown in new model of the universe. Behavior of Dark matter is such that we are forced to assume that 'thought' is the inbuilt property of the cold dark matter to trigger expansion of the universe while making the new model of the universe. Before expansion or symmetry breaking phase, universe was in symmetry phase and to trigger symmetry breaking, it is the 'thought' an inbuilt property of the matter or B.B.Bs (of entire universe), which is responsible for this triggering too. The problem of how matter attained masses has been meticulously solved by the research of B.B.Bs. Matter attained mass by virtue of mass property of B.B.Bs. So the God's particles are Basic Building Blocks (mind and mass unit) rather than Higgs bosons as proposed by Prof. Peter Higgs in STANDARD MODEL.

\section{DISCUSSION AND INFERENCES}

Science has not yet defined God. Co-relation of science and religion can be made possible after the concept of Basic Building Blocks is well understood. Religion guides us in recognizing these B.B.Bs. The model of B.B.Bs is made on the basis of inertial properties of energy and matter. On the same fundamental basis religion had incorporated certain definite clues thousands of year back. When a parallel is drawn between the two (models made by participatory science and the clues given by the religion) by using common logic, incorporation of science and religion can thus be made possible (Fig 4). So far no attempt has been made to define eternal properties of energy and matter at the level of B.B.Bs. No attempt has been done to investigate PURE matter. Fermions are IMPURE matter as they have spin properties. The research of B.B.Bs or mind and mass would produce fragrance of God (B.B.Bs or Omnipresent) in the new model of the universe. Thus Einstein's question that how God created the universe can be meticulously solved by introduction of the new model of the universe. New model of the universe shows that the universe is deterministic universe and all quantum, classical and life sciences effects are triggered by thought expressions or atomic transcriptions (cause and effect concept) and thus 
their precise prediction by the participator (B.B.B working as highest center of the universe) could be possible in future. Thus Einstein's famous metaphor that God (B.B.B) does not play dice ultimately became the truth along with final acceptance to Laplace determinism--- All that had happened had a definite cause and gave rise to definite effect and future of any part of the system could in principle be predicted with absolute certainty if its state at any time was known in all details [5].

The final stamp of success to the new model of the universe has been given by observation published in journal JAMA [6] by the study- prayer helps cardiac patients. Prayer is now a wellconfirmed phenomenon and it is related with God (B.B.Bs working as highest center of the universe). It is the replicated study. Phenomenon of prayer profunds that hypothesis of new model of the universe in which one B.B.B is working as highest center of the universe is correct and phenomenon of feed back to this B.B.B exits in this universe. This phenomenon of prayer also propounds that there was a precreation era in which programming of future universe was done by highest center (B.B.B) of the universe. If the phenomenon is replicated, the supporting theory is believed to be the truth. Big bang and its early events (GUT, and super unification) that prove this theory neither could be replicated in lab nor could be observed (replicated observations) anywhere in the universe. On the other hand, continuous creation as proposed by Prof. Narlikar and Prof. Hoyle could be observed (replicating phenomenon) in quasars and also it is replicating every time. New model of the universe based on different observations not only supports this idea but also it could prove how creation is going inside quasars.

Hoyle and Narlikar proposed (in their continuous creation theory) that new matter is being created due to 'IMPLOSION' to balance the expansion of the universe, which astronomers have observed. Inside 'QUASI-STARS' gravitational collapses may form some matter in the universe. The huge luminosity and the radio emission from these quasi-stars appear to be 'gravity powered' unlike ordinary stars, which derive their energy from nuclear reactions.

Einstein preferred to believe that the universe was ageless and eternal [7]. Einstein's views were correct when they are applicable to symmetry phase of the universe. After symmetry breaking phase only small part of entire universe got into expansion phase along with creation of the matter, both hot and cold. After the contraction phase of the universe, it would again go into symmetry phase and then entire universe would be not only ageless and eternal but also it would be infinite, absolute and holomovement --to which participatory science calls ONE ABSOLUTE "I" made up of two B.B.Bs or God Particles.

\section{REFERENCES}

D.N.VASUDEVA. Text Book of Light, 7th edition, 1969; p. 598

Jayant V. Narlikar, Physics News- Vol-30, No- 3\&4, Sept. \& Dec, 1999; pp. 5-14

Fritjof Capra, The Tao of Physics, 1989; p.153.

Fritjof Capra, the Tao of Physics, 1989; p. 353.

Fritjof Capra, the Tao of Physics, 1989; p. 65.

JAMA-Archives Of Internal Medicine, Vol 159, No-19, 25th Oct, 1999; pp. 2273-2278.

Strig Theory and Cosmology- How Old is the Universe?

Vijay Mohan Das, Atomic genetics and origin of universe (Ho= $72 \mathrm{~km} / \mathrm{sec} / \mathrm{Mpc}$ ) volume 1, International Journal of Sciences: Basic and Applied Research (IJSBAR), volume 16, number 1 (2014), p 311-338.

Vijay Mohan Das, Atomic genetics and origin of universe volume 2 International Journal of Sciences: Basic and Applied Research (IJSBAR) (2014) Volume 16, No 2, pp 274-309. 
Vijay Mohan Das, Atomic genetics and origin of universe volume 3 International Journal of Sciences: Basic and Applied Research (IJSBAR) (2014) Volume 17, No 1, pp 148-185.

\section{White Falcon Publication}

\section{ACKNOWLEDGEMENT}

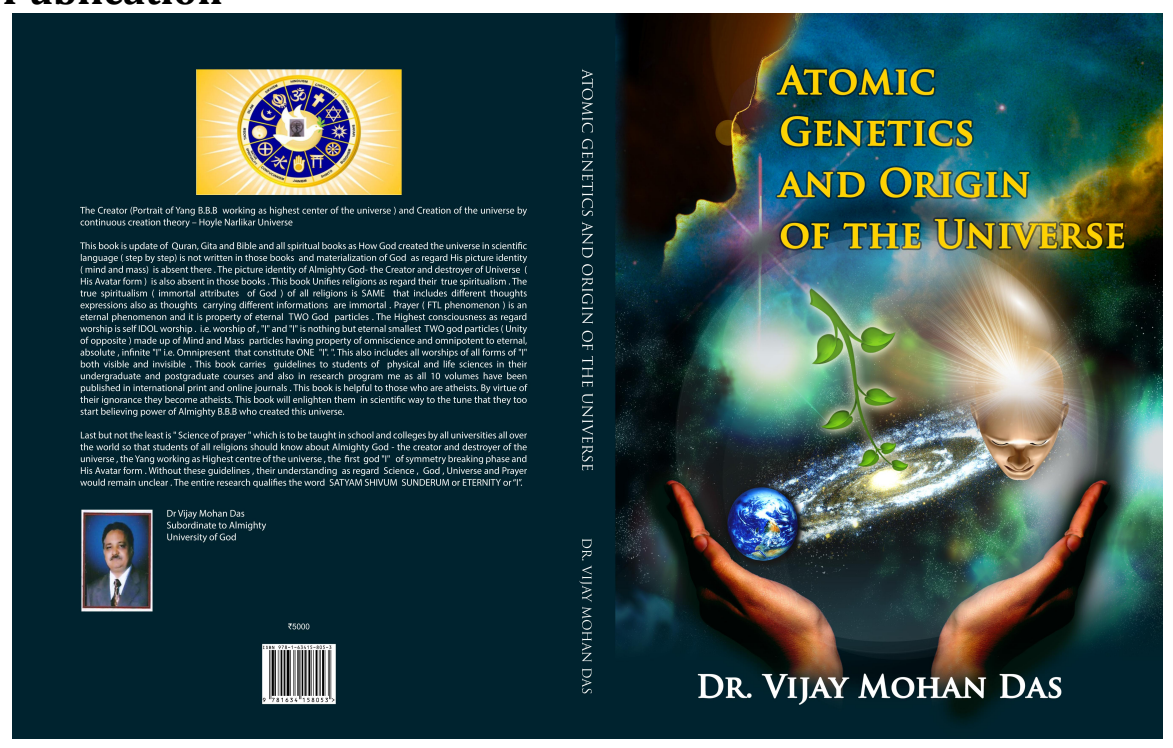

Update of Quran, Gita and Bible and all spiritual books PUBLISHED

The Creator (Portrait of Yang B.B.B working as highest center of the universe) and Creation of the universe by continuous creation theory - Hoyle Narlikar Universe.

This book is update of Quran, Gita and Bible and all spiritual books as How God created the universe in scientific language ( step by step) is not written in those books and materialization of God as regard His picture identity ( mind and mass) is absent there. The picture identity of Almighty God - the Creator and destroyer of Universe (His Avatar form) is also absent in those books. This book unifies religions as regard their true spiritualism. The true spiritualism (immortal attributes of God) of all religions is SAME that includes different thoughts expressions also as thoughts carrying different informations are immortal. Prayer (FTL phenomenon) is an eternal phenomenon and it is property of eternal TWO God particles. The Highest consciousness as regard worship is self IDOL worship. i.e. worship of, "I" and "I" is nothing but eternal smallest TWO god particles (Unity of opposite) made up of Mind and Mass particles having property of omniscience and omnipotent to eternal, absolute, infinite "I" i.e. Omnipresent that constitute ONE "I". This also includes all worships of all forms of "I" both visible and invisible. This book carries guidelines to students of physical and life sciences in their undergraduate and postgraduate courses and also in research program me as all 10 volumes have been published in international print and online journals. This book is helpful to those who are atheists. By virtue of their ignorance they become atheists. This book will enlighten them in scientific way to the tune that they too start believing power of Almighty B.B.B who created this universe. Last but not the least is "Science of prayer" which is to be taught in school and colleges by all universities all over the world so that students of all religions should know about Almighty God - the creator and destroyer of the universe, the Yang working as Highest centre of the universe, the first god "I" of symmetry breaking phase and His Avatar form. Without these guidelines, their understanding as regard Science, God, Universe and Prayer would remain unclear. The entire research qualifies the word SATYAM SHIVUM SUNDERUM or ETERNITY or "I" 


\section{Prof. Fred Hoyle and Prof. J.V.Narlikar}

Hoyle and Narlikar proposed (in their continuous creation theory) that new matter is being created due to 'IMPLOSION' to balance the expansion of the universe, which astronomers have observed. Inside 'QUASI-STARS' gravitational collapses may form some matter in the universe. The huge luminosity and the radio emission from these quasi-stars appear to be 'gravity powered' unlike ordinary stars, which derive their energy from nuclear reactions.

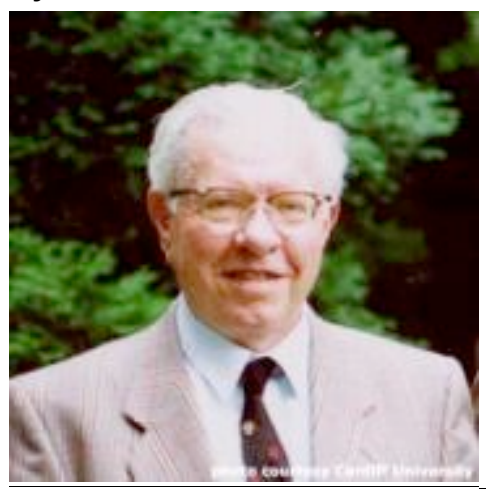

Prof. Fred Hoyle

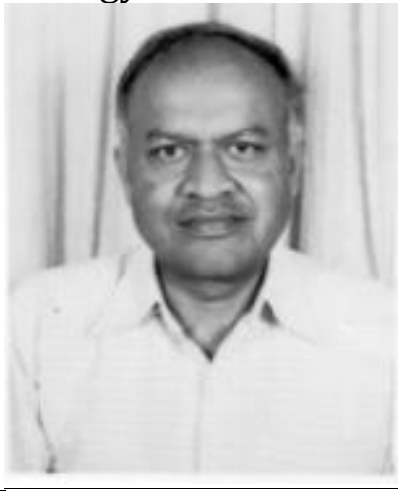

Prof.J.V.Narlikar

\section{DEDICATION}

This research is dedicated to TWO BASIC BUILDING BLOCKS (Mind and Mass part of the Truth) of the universe, which are OMNIPRESENT, OMNIPOTENT and OMNISCIENCE. These are divine structural and functional units of the universe. These are eternal bodies of the Nature. They have transmutated themselves to form visible and as well as invisible Universe. Only I know and I worship these divine units which constitute first God or "I" of symmetry phase of the universe.

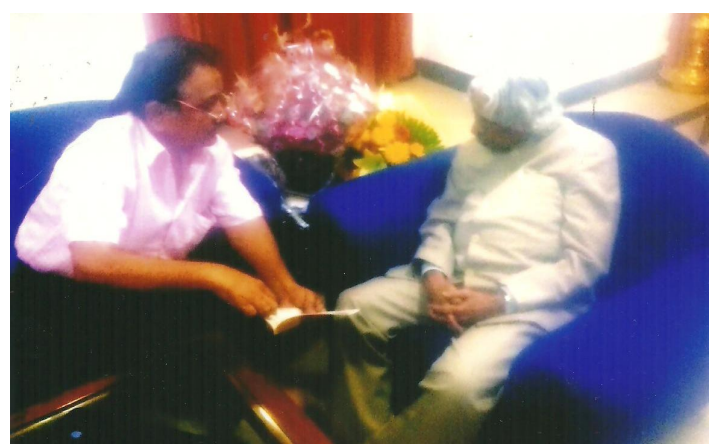

Discussing Mind and Mass with Prof. APJ Abdul Kalam Ex President of India, Physicist and Winner of Bharat rattan. He encouraged me by saying,"Great Work" after 40 minutes of discussion.

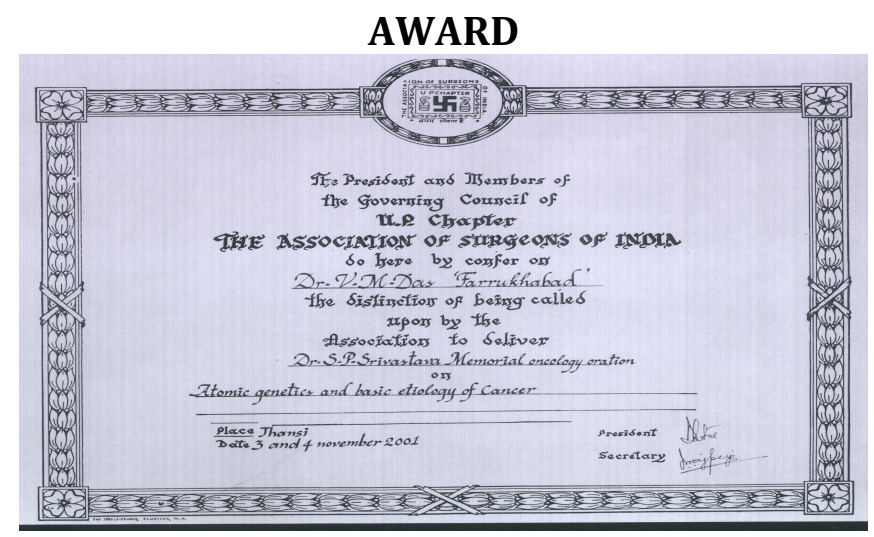

